\title{
Structure of Plasticity in Human Sensory and Motor Networks Due to Perceptual Learning
}

\author{
Shahabeddin Vahdat, ${ }^{1,2 *}$ Mohammad Darainy, ${ }^{1 *}$ and David J. Ostry ${ }^{1,3}$ \\ ${ }^{1}$ McGill University, Montreal, Quebec H3A 1B1, Canada, ${ }^{2}$ Functional Neuroimaging Unit, University of Montreal, Quebec H3W 1W5, Canada, and ${ }^{3}$ Haskins \\ Laboratories, New Haven, Connecticut 06511
}

As we begin to acquire a new motor skill, we face the dual challenge of determining and refining the somatosensory goals of our movements and establishing the best motor commands to achieve our ends. The two typically proceed in parallel, and accordingly it is unclear how much of skill acquisition is a reflection of changes in sensory systems and how much reflects changes in the brain's motor areas. Here we have intentionally separated perceptual and motor learning in time so that we can assess functional changes to human sensory and motor networks as a result of perceptual learning. Our subjects underwent fMRI scans of the resting brain before and after a somatosensory discrimination task. We identified changes in functional connectivity that were due to the effects of perceptual learning on movement. For this purpose, we used a neural model of the transmission of sensory signals from perceptual decision making through to motor action. We used this model in combination with a partial correlation technique to parcel out those changes in connectivity observed in motor systems that could be attributed to activity in sensory brain regions. We found that, after removing effects that are linearly correlated with somatosensory activity, perceptual learning results in changes to frontal motor areas that are related to the effects of this training on motor behavior and learning. This suggests that perceptual learning produces changes to frontal motor areas of the brain and may thus contribute directly to motor learning.

Key words: motor adaptation; perceptual learning; plasticity; resting-state fMRI; sensorimotor network

\section{Introduction}

Perceptual learning may play a significant role in motor learning, particularly early in learning, where somatosensory goals of movement are poorly defined. Learning the feel of a good golf swing or learning to speak in a foreign language requires the progressive adjustment of motor commands and the development of a sensory plan, a trajectory of desired sensory values, that regulates the generation of movement. However, in situations such as these where sensory and motor systems are active in tandem, the source of the neural and behavioral changes with learning is uncertain. Changes could be attributable to motor learning driving sensory change, to perceptual learning driving movement, or the two in combination. In the present article, we segregate perceptual and motor learning in time to focus on the neural correlates of perceptual learning in the motor system. We find that perceptual learning results in changes to motor areas of the brain. These changes are present after removing effects in

\footnotetext{
Received Oct. 7, 2013; revised Dec. 5, 2013; accepted Jan. 3, 2014.

Author contributions: S.V., M.D., and D.J.O. designed research; S.V. and M.D. performed research; S.V. contributed unpublished reagents/analytic tools; S.V. and M.D. analyzed data; S.V., M.D., and D.J.O. wrote the paper.

This research was supported by National Institute of Child Health and Human Development Grant R01 HD075740 and by Le Fonds Québecois de la Recherche sur la Nature et les Technologies (Quebec). We thank Brian Hynes (Hybex Innovations, Montreal, QC, Canada) for the design and construction of the MR-compatible sensory testing apparatus.

*S.V. and M.D. contributed equally to this work.

Correspondence should be addressed to David J. Ostry, Department of Psychology, McGill University, 1205 Dr. Penfield Avenue, Montreal, QC H3A 1B1, Canada. E-mail: david.ostry@mcgill.ca.

DOI:10.1523/JNEUROSCI.4291-13.2014

Copyright $\odot 2014$ the authors $\quad 0270-6474 / 14 / 342451-13 \$ 15.00 / 0$
}

motor areas that are linearly related to those in sensory systems. Our analysis thus suggests that perceptual learning may produce changes to motor areas of the brain that may be functionally independent of those observed in sensory systems.

There are recent studies that indicate an effect of perceptual training on motor learning. In studies of limb motor control and speech motor learning, Darainy et al. (2013) and Lametti et al. (2012) show that brief periods of reinforced perceptual training have durable effects on the rate and extent of motor learning. Rosenkranz and Rothwell (2012) find that somatosensory discrimination training increases primary motor cortex excitability and improves measures of motor learning. Wong et al. (2012) report that passive movement of the arm increases the extent of motor learning. Perceptual stimulation and learning thus benefit movement and motor learning. But to understand the neural basis of these effects, it is necessary to separate those changes to motor areas that can be predicted from cortical sensory activity from those that are independent of activation in sensory areas of the brain.

Here we test the idea that somatosensory perceptual learning leads to persistent changes to motor areas of the brain that cannot be explained by somatosensory changes alone. Subjects underwent fMRI scans of the resting brain before and after a somatosensory perceptual discrimination task that focused on training subjects to correctly identify the position of their arm in space. We examined changes in functional connectivity (FC) in the sensorimotor network that varied with subsequent movement and motor learning. We tested for changes in connectivity that were 

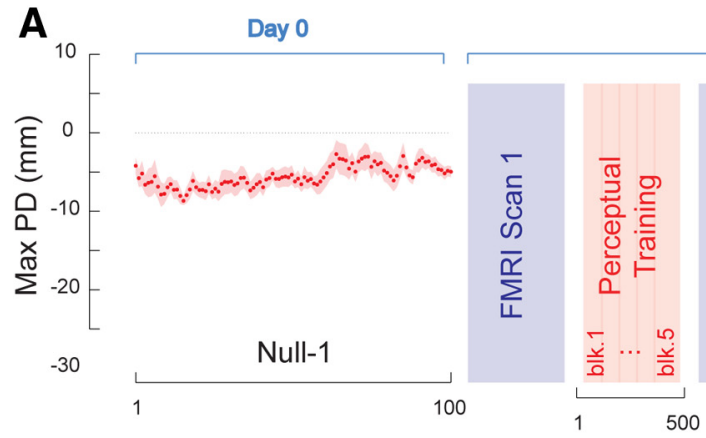

500
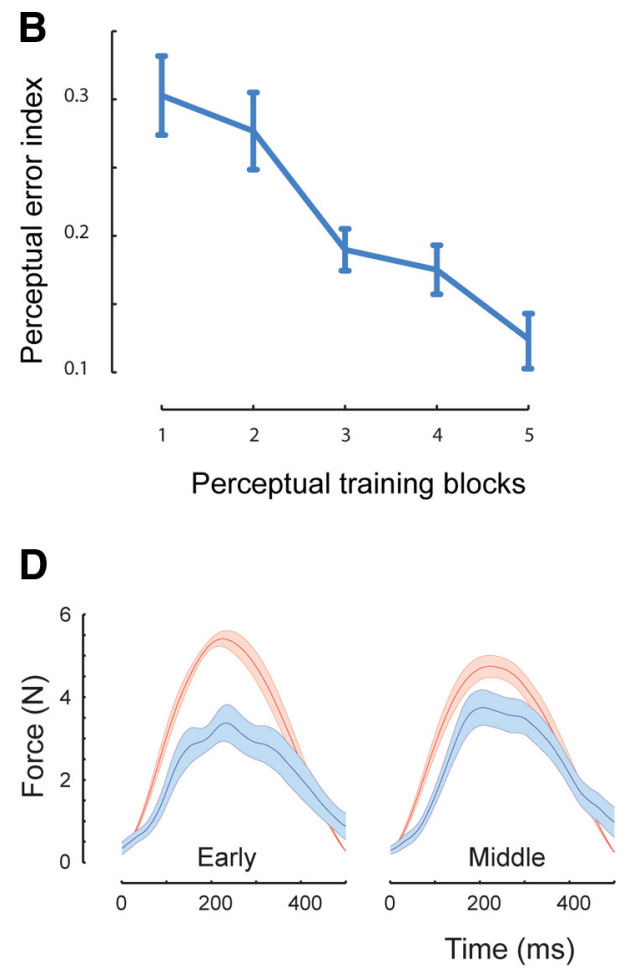

C
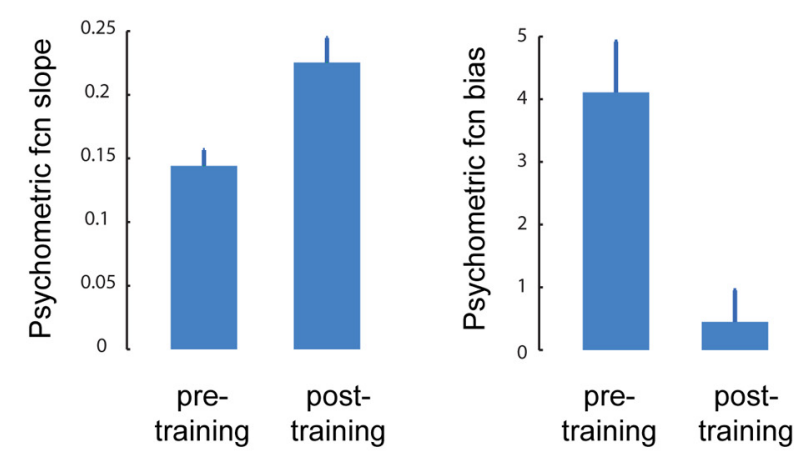

E

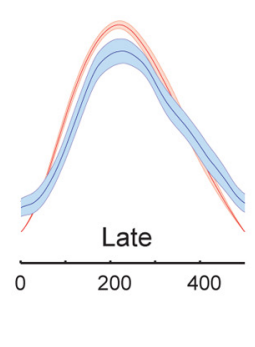

Day 1

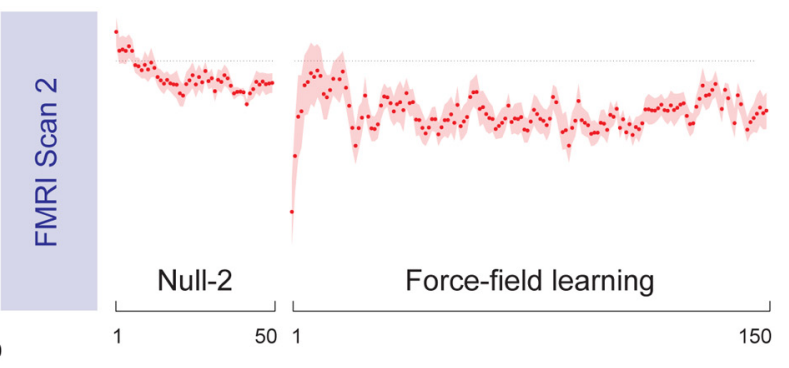

Trial number

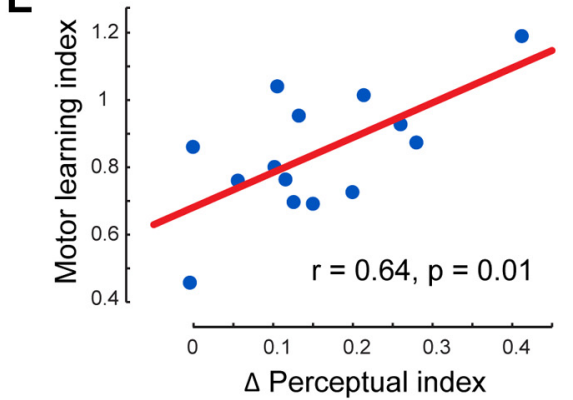

Figure 1. Somatosensory perceptual learning benefits both perceptual and motor behaviors. $A$, Experimental sequence, fMRI scanning sessions, and average lateral movement deviation in different phases of the experiment. Shaded area shows SEM. B. The PI improves over the course of somatosensory training (mean over subjects \pm SEM). Lower PI values indicate decreased perceptual classification error. C, The perceptual boundary (psychometric function bias, right) and acuity (psychometric function slope, left) change over the course of somatosensory training. Bar plots show the average performance in the first (block 1 ) and final (block 5 ) blocks of perceptual training (mean over subjects \pm SE). $\boldsymbol{D}$, Motor learning as reflected in lateral force production in channel trials early, midway, and late in force-field learning. Lateral applied force profiles are shown in blue averaged across subjects. The red curves show the ideal force profile that subjects would need to exert to fully compensate for the force-field perturbation. $\boldsymbol{E}$, Subjects who learned more during perceptual training (as measured by $\Delta \mathrm{PI}$ ) subsequently exhibited greater amounts of motor learning (as measured by FI). Each point represents one subject.

uncorrelated with activity in the somatosensory network. For this purpose, we used a model of perceptual discrimination learning in combination with a partial correlation procedure to remove the effects on observed connectivity patterns of activity in earlier stages of the somatosensory network. We found that even after the correlates of activity in somatosensory areas were removed, perceptual training resulted in changes to connectivity in frontal motor areas of the brain and cerebellum. Thus, a portion of the change in motor areas of the brain that occurs in learning skilled movements, that is, when perceptual and motor learning occur in tandem, may actually be the product of perceptual learning.

\section{Materials and Methods}

Subjects and experimental design. A total of 14 right-handed subjects (9 male, 5 female), $20-43$ years of age, participated in a combined perceptual training and brain-imaging experiment, which included three behavioral and two scanning sessions. All subjects were briefed on the experiment and signed a written consent form. The Institutional Review Board of McGill University approved all the experimental procedures.

The behavioral sessions involved a number of different tasks in which subjects were seated in front of a $2 \mathrm{df}$ robotic arm (In Motion2, Interactive Motion Technologies) and held the handle of the robot with their right hand. The seat height was adjusted for each subject separately to have $70^{\circ}$ of shoulder abduction. An air sled was used to support the arm, and seat straps restrained the trunk. The position of the target and the subject's hand were projected on a semi-silvered mirror that was placed just below eye level. The mirror blocked the sight of the arm and the robot handle. Optical encoders recorded the position of the hand $\left(0.0055^{\circ}\right.$ resolution, Virtual Absolute Encoder, Gurley Precision Instruments). Applied forces were measured using a force-torque sensor [0.028 N resolution; Gamma-30-100, ATI Industrial Automation] that was mounted below the robot handle. 
The experimental sequence is shown in Figure $1 A$. On Day 0, subjects came to the laboratory and were briefed on the psychophysical procedure and also performed 100 reaching movements in a null-field condition to establish a movement baseline. In null-field trials, the robot applied no force to the subject's hand. Twenty-four hours later, on Day 1, subjects came to the Montreal Neurological Institute (MNI) imaging facility. A first scanning sequence took $\sim 30 \mathrm{~min}$. Subjects then moved to the laboratory for somatosensory training (see below). Afterward, they returned to the imaging facility for a second set of scans that lasted $\sim 1 \mathrm{~h}$. Finally, they returned to the laboratory and completed 50 reaching movements under null-field conditions followed by 150 trials in a counterclockwise force field that pushed the hand to the left in proportion to instantaneous hand velocity (Fig. 1A).

Sensory and motor psychophysical procedures. Subjects made reaching movements from a start position that was $\sim 25 \mathrm{~cm}$ from subject's chest along the body midline to a target that was $15 \mathrm{~cm}$ from the start in the sagittal plane. Circles represented the movement start and end points. Subjects were instructed to make straight movements and to finish each movement in $\sim 700 \mathrm{~ms}$. Visual feedback on movement speed was provided. A small yellow circle was used to indicate the hand position. All visual feedback (target and hand position) was removed as soon as the subject left the start position. Feedback reappeared at the end of movement. At the end of the trial, the robot moved the hand back to the start position, without visual feedback.

The somatosensory training procedure, which took place on the second day of the experiment, involved five blocks of 100 trials each, in which the robot passively moved the arm outward on one of a set of fan-shaped trajectories that deviated to the right or the left of the body midline by up to $12^{\circ}$. The sight of the arm was blocked throughout this part of the experiment. Hence, subjects could only use somatosensory information to identify movement direction. On each trial, subjects were required to judge whether the arm had been moved to the right or left (oral response). In the last three blocks of sensory training, the experimenter provided oral feedback regarding the correctness of subject's response (by answering yes or no). In the first two blocks, feedback on judgment accuracy was withheld to provide a baseline measure of perceptual function before the training trials with feedback.

In the perceptual training sequence, the robot passively moved the subject's arm outward along 10 straight trajectories with a fan-shaped distribution that were equally spaced to the right or the left of the midline (Darainy et al., 2013). The passive movements had bell-shaped velocity profiles and were $15 \mathrm{~cm}$ in length. Lateral deviations of $12^{\circ}, 8^{\circ}, 5^{\circ}, 3^{\circ}$, and $1.5^{\circ}$ in both directions relative to midline were used for purposes of perceptual training. The above angles were tested $6,8,10,12$, and 14 times each, again in both directions, in each block of 100 trials. Subjects were instructed not to resist the action of the robot so as to minimize any active motor outflow during the sensory training procedure. This was subsequently verified using the recorded force exerted by subjects to the handle during the passive movement training (see Results).

The force-field trials involved a counterclockwise load that pushed the subject's hand to the left in proportion to hand velocity. The force field is given in Equation 1, as follows:

$$
\left[\begin{array}{l}
f_{x} \\
f_{y}
\end{array}\right]=\left[\begin{array}{cc}
0 & -15 \\
15 & 0
\end{array}\right]\left[\begin{array}{l}
v_{x} \\
v_{y}
\end{array}\right],
$$

where $x$ and $y$ are lateral and sagittal directions, respectively, $f_{x}$ and $f_{y}$ are the force (in newtons) applied by the robot, and $v_{x}$ and $v_{y}$ are hand velocity (in meters per second) in Cartesian coordinates. The units of the gain coefficient are newton second per meter.

On five of the force-field learning trials $(15,85,135,139$, and 143), the lateral deviation of a subject's hand was resisted by the robot, so as to restrict a subject's movement to a straight line connecting the start and target points ("channel trials"). The stiffness and viscosity of the channel walls were set to $5000 \mathrm{~N} / \mathrm{m}$ and $50 \mathrm{~N} \mathrm{~s} / \mathrm{m}$. The lateral forces that subjects applied to the channel walls provide a measure of motor learning.

Brain-imaging procedures. All data were acquired using a 3 tesla Siemens Trio MR scanner at the MNI. Whole-brain functional data were acquired using a T2*-weighted EPI sequence (32-channel phased-array head coil; resolution, $3 \mathrm{~mm}$ isotropic; 47 slices; $64 \times 64$ matrix; TE, 30 $\mathrm{ms}$; TR, $2500 \mathrm{~ms}$; flip angle, $90^{\circ}$; generalized autocalibrating partially parallel acquisition with an acceleration factor of 2). The functional images were superimposed on a T1-weighted anatomical image (resolution, $1 \mathrm{~mm}$ isotropic; 192 slices; $256 \times 256$ matrix).

In the first fMRI session, two 7 min functional scans of the resting brain were acquired with the eyes closed. High-resolution anatomical images of the brain were obtained between the two resting-state scans. The second fMRI session followed the same procedure. After the final resting-state scan in the second session, subjects completed two additional 6 min functional scans, each using an event-related passive arm movement paradigm similar to that used for the somatosensory discrimination training. The passive movement data were used as localizers to obtain seed voxels for the resting-state functional connectivity analyses. In the localizer task, subjects closed their eyes and held the handle of a Plexiglas magnet-compatible device (Hybex Innovations; Fig. 2A) while lying in the supine position in the fMRI scanner. The experimenter moved the subject's right arm forward and backward in a coronal plane. The shoulder was not restrained, and hence the device produced movement at both joints. The movement amplitude was $12 \mathrm{~cm}$. The arm was moved at randomly selected angles relative to the body midline that were drawn from the following set $\left[-30^{\circ},-20^{\circ},-10^{\circ}, 10^{\circ}, 20^{\circ}\right.$, and $30^{\circ}$. In total, there were 58 passive movements, each lasting $\sim 2 \mathrm{~s}$. The head motion artifact was minimized by using cushions to stabilize the head. The mean $( \pm S D)$ absolute value of head displacement averaged across subjects and runs was $0.20 \pm 0.16 \mathrm{~mm}$, estimated using MCFLIRT in FSL. The maximum displacement was $1.5 \mathrm{~mm}$, which suggests a minimal motion artifact due to passive movement.

The interval between consecutive trials was randomly varied between 9 and $12 \mathrm{~s}$, during which time the angle for the following trial was set by the experimenter. The design of the apparatus minimized the movement of the cone-shaped handle as it was being repositioned between trials. Subjects were instructed to indicate whether their arm was moved rightward or leftward and respond accordingly with their left index finger, using a two-button fiber optic response pad (Current Designs), as soon as the passive movement stopped. The response times were recorded in reference to the fMRI volume acquisition. The timing of passive movements was synchronized using visual stimuli that were visible only to the experimenter, and specified the onset and duration of movement with respect to the fMRI volume acquisition. As we did not have precise control over the end of passive movements, the timing of each trial in our eventrelated analysis lasted up to $0.3 \mathrm{~s}$ before the subject's button press. This included the time for the passive movement (on average, $2 \mathrm{~s}$ ) and the delay time before the response (on average, $0.4 \mathrm{~s}$ ). Furthermore, we added the derivative of the event-timing signal to the general linear model to account for inaccuracy in the timing of the onset and end time of each movement. Nevertheless, some variability due to inaccurate timing remains unaccounted for.

Behavioral data analysis. The maximum perpendicular deviation (PD) of the hand from a line connecting start and end positions was used for analysis purposes. This measure provided a quantitative estimate of movement straightness that was used to examine motor performance and learning. We calculated the average of PD in the final 50 trials of the first and the second null-field conditions $\left(\overline{\mathrm{PD}}_{\text {pre }}\right.$ and $\overline{\mathrm{PD}}_{\text {post }}$, respectively) for each subject separately. We tested for differences in PD between these null-field values using a repeated-measures ANOVA. Furthermore, to quantify the motor-related benefits of perceptual training, we defined a motor index (MI) on a per-subject basis as the change in average PD from pretraining to post-training null-field conditions, as follows:

$$
\mathrm{MI}=\overline{\mathrm{PD}}_{\mathrm{pre}}-\overline{\mathrm{PD}}_{\text {post }}
$$

We also quantified motor learning by measuring the total lateral force in three channel trials that were recorded late in the training sequence (averaged over trials 135,139 , and 143), divided by the ideal total force that was needed to fully compensate for the force field. A force index (FI) was defined as follows: 


$$
\mathrm{FI}=\frac{\int_{0}^{T} f_{x}(t) d t}{\int_{0}^{T} 15 v_{y}(t) d t},
$$

where $f_{x}(t)$ is the lateral force applied by the subject, $v_{y}(t)$ is the velocity in the direction of movement, and 15 is the coefficient relating applied force to hand velocity (Eq. 1).

We estimated subject's perception of the boundary between left and right by using the method of constant stimuli. A logistic function was fit to each subject's entire set of lateral deviations and associated binary (right/left) responses (Ostry et al., 2010). The $50 \%$ point of the fitted psychometric function gave the perceptual boundary. The distance between the 25th and 75th percentiles served as a measure of perceptual acuity. An aggregate measure of perceptual classification accuracy, a perceptual index (PI), was obtained by computing the difference between the best possible performance and the actual false-positive and true-positive values.

$$
\mathrm{PI}=\sqrt{\mathrm{FP}^{2}+(1-\mathrm{TP})^{2}} .
$$

In this equation, $\mathrm{FP}$ is the proportion of falsepositive responses, the number of incorrect responses for rightward deflections divided by the total number of rightward movements. TP is the proportion of true-positive responses. So, 1 - TP is the proportion of misses, the number of incorrect responses for leftward deflections divided by the total number of leftward movements. A value for PI was obtained for each block of perceptual training. The change in PI from the first to the final block of perceptual training $\left(\Delta \mathrm{PI}=\mathrm{PI}_{\text {block1 }}\right.$ $-\mathrm{PI}_{\text {block5 }}$ ) was used as a measure of perceptual improvement.

FMRI data analysis. Image preprocessing was performed using the FSL software package (Beckmann et al., 2003), using the same preprocessing pipeline as described previously (Vahdat et al., 2011). We used both seed-based functional connectivity analysis and independent components analysis (ICA) to study the structural and temporal characteristics of the perceptual learning memory trace.

Partial correlation applied to seed-based functional connectivity analysis. We conducted a seed-based functional connectivity analysis based on a model of the perceptual decision-making sequence derived from electrophysiological work on nonhuman primates (Romo and Salinas, 2003; de Lafuente and Romo, 2006; Romo and de Lafuente, 2013; Fig. 2B). The sequence shown in Figure $2 B$ was constructed using propagation delays through the sensorimotor network (de Lafuente and Romo, 2006; Hernández et al., 2010). This simple model fits with the idea that there is an ordering to the transformation of information from a pure sensory signal to a motor action required in perceptual discrimination. We defined nine regions of interest (ROIs) that we have used in conjunction with this model based on the somatosensory localizer task performed in the scanner (Table 1). These regions are as follows: primary somatosensory cortex (left BA1, BA2, BA3b, and right BA1/2), second somatosensory cortex within the parietal operculum (left SII), ventral premotor cortex (left PMv), dorsal premotor cortex (left PMd), supplementary motor area (SMA), and primary motor cortex (left M1). The seed locations within each of these cortical areas were identified using the peaks of activity from an event-related analysis of the BOLD response during the passive movement/somatosensory discrimination task, as described ear-

B data.

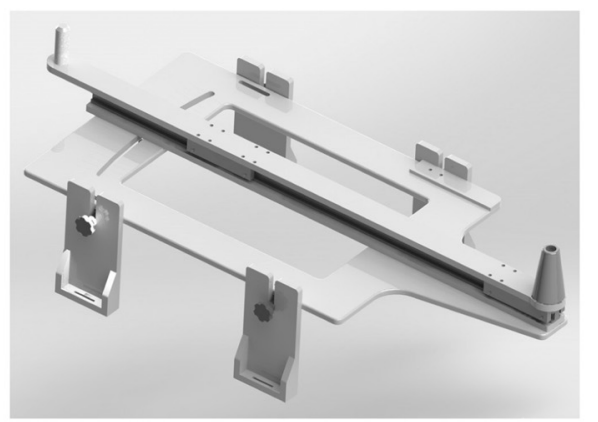

Figure 2. A, Drawing of MR-compatible device that was used in the scanner to passively move the subject's arm. Subjects held the device (shown to the right), while the experimenter moved the handle located at the left end in the perceptual hierarchy. C, Processing pipeline showing different computational steps in dynamic ICA analysis of resting-state

Table 1. Activation peaks from a somatosensory localizer task performed in the scanner

\begin{tabular}{llrlll}
\hline & & \multicolumn{3}{l}{ MNI coordinates } & \\
\cline { 3 - 4 } R01 & Anatomical label & $x$ & $y$ & $z$ & z-value \\
\hline BA2 & Left primary somatosensory cortex BA2 & -48 & -22 & 38 & 7.0 \\
BA3b & Left primary somatosensory cortex BA3b & -20 & -34 & 66 & 5 \\
BA1 & Left primary somatosensory cortex BA1 & -56 & -14 & 42 & 5.1 \\
BA2/R & Right primary somatosensory cortex BA2 & 50 & -28 & 56 & 4.8 \\
SII & Left secondary somatosensory cortex, OP1 & -38 & -26 & 16 & 6.9 \\
PMv & Left ventral premotor cortex, BA6 & -42 & -10 & 48 & 5.2 \\
PMd & Left dorsal premotor cortex, BA6 & -40 & -16 & 62 & 5.0 \\
SMA & supplementary motor area BA6 & 0 & -10 & 52 & 7.8 \\
M1 & Left primary motor cortex BA4a & -10 & -30 & 74 & 6.0 \\
\hline
\end{tabular}

The table shows seed coordinates in MNI space (given in millimeters), their anatomical labels, and their $z$-values from the event-related analysis.

lier. Conducting this somatosensory localizer task in the scanner ensured that the selected seed voxels corresponded somatotopically to areas activated by subjects' arm afferents and the perceptual decision-making task (Table 1). By including BA1/2 and BA3b, we ensure that we have selected areas that receive both proprioceptive and cutaneous information in the context of the present perceptual training task. Area 3a was intentionally excluded from these analyses because of its proximity to BA4; hence, the difficulty in distinguishing between motor and somatosensory activations. 
We defined a standard spherical mask (radius $=6 \mathrm{~mm}$ ) around each seed in standard space. We resampled this mask first to the T1-weighted structural image of each subject and from there to the low-resolution functional space of that subject. For each subject, the average time course of the BOLD signal within the transformed mask during the resting-state scans was calculated. The mean BOLD time course of each ROI was used as a predictor in a per-subject GLM to assess the functional connectivity of that ROI with every other voxel in the brain.

A simple technique based on the notion of partial correlation (Marrelec et al., 2006) was used in conjunction with the model in Figure $2 B$ to investigate the hierarchy of information processing within the sensorimotor system following perceptual training. To obtain a brain connectivity map between area $Y$ and the rest of brain when the contributions of areas $X 1, X 2, \ldots X n$ are eliminated (i.e., conditional correlation $\operatorname{Corr}(Y \mid X 1$, $X 1, \ldots X n$ ), we added the time series of the conditioning areas $X 1, X 2, \ldots X n$ as confounds in the subject-level GLM analysis with the time series of $Y$ as the regressor of interest (orthogonalized with respect to all confound regressors). This is mathematically equivalent to subtracting and removing mutual dependencies of the conditioning areas from the connectivity map of area $Y$. For each individual, a separate multiple regression analysis was performed using the time series of nuisance signals described in Vahdat et al. (2011) (including averages of white matter, CSF, and global signals) plus the time series of conditioning areas as confound regressors, with the time series of the ROI as the regressor of interest. This analysis was conducted separately for the prelearning and postlearning scans.

We performed the conditional functional connectivity analysis for the four different levels of perceptual processing shown in the model described in Figure $2 B$. Level 1 gives the pattern of connectivity for the regular seed-based analysis. That is, it assesses connectivity associated with seeds in primary somatosensory areas. Level 2 uses SII and PMv as seeds, with the signal correlates of level 1 subtracted out. Level 3 has seeds in PMd and SMA with the effects of all areas in levels 1 and 2 removed. Finally, level 4 has M1 as the seed region, with the effects of all areas earlier in the sequence (BA1, BA2, BA3b, SII, PMv, PMd, SMA) removed.

This analysis produced maps of all voxels that were positively or negatively correlated with the mean time course of an ROI, with the signal correlates of all the preceding steps in the perceptual processing hierarchy removed. The subject-level regression analysis was followed by between-subjects analyses that were performed using a mixed-effects model (FLAME) implemented in FSL (Beckmann et al., 2003). As in Vahdat et al. (2011), we used behavioral factors (MI, PI, and FI) as regressors to obtain a weighted average of the difference between scans (post-training compared with pretraining conditions). Thus, instead of using the binary contrast Scan $2-S \operatorname{can} 1$ as the contrast of interest in the GLM, we used a graded variable that was based on each subject's perceptual training performance. Corrections for multiple comparisons at the cluster level were performed using Gaussian random field theory (minimum $z>2.7$; cluster significance, $p<0.05$, corrected). To correct for multiple ROIs, we identified as statistically significant those clusters that had a probability level of better than $p=0.05 / 9$ ( 9 is the number of ROIs). This between-subjects analysis produced thresholded $z$-score maps of activity associated with each ROI.

We then examined the correspondence between changes in the behavioral measures and changes in functional connectivity from pretraining to post-training conditions. We constructed a vector for each connection between an ROI and target cluster whose elements were each subject's change in functional connectivity $(\Delta \mathrm{FC})$ from pretraining to posttraining sessions. This vector was correlated with a vector of associated behavioral measures. In the present study, we report only those connections whose change in FC was reliably correlated with behavior $(p<$ 0.05).

Temporal dynamics analysis using shared and specific independent component analysis. We also used a recently developed ICA method, the shared and specific independent component analysis (SSICA; Vahdat et al., 2012), to investigate the temporal dynamics of brain networks that are preferentially activated during the post-training condition. SSICA is used to systematically perform between group/condition comparisons in the ICA framework. This method is particularly useful in resting-state conditions when there is no temporal constraint imposed by the task design. SSICA automatically extracts those components that represent a significant difference in functional connectivity across conditions (the so-called specific components corresponding to each condition). Here, we first applied the SSICA on the resting-state dataset to identify those brain networks that are common to the pretraining and post-training conditions and those that are specific to either condition. The focus of the analyses presented below is on those networks that are specific to one condition or the other, as these reflect the changes in brain networks as a result of perceptual training. In these analyses, the resting-state BOLD signal is preprocessed using the following steps: motion correction, highpass temporal filtering, slice timing correction, spatial smoothing $(6 \mathrm{~mm}$ Gaussian kernel), registration to the MNI standard space, and downsampling to $4 \mathrm{~mm}$ isotropic space. The dimension of subject-level functional data for each session was reduced from 330 ( 2 runs $\times 165$ volumes per run) to 50 as part of SSICA preprocessing. To extract the shared and specific components across conditions, all the reduced resting-state fMRI sessions ( 28 sessions: 14 subjects $\times 2$ conditions per subject) belonging to both pretraining and post-training conditions were time concatenated and fed to the SSICA algorithm. We extracted 30 networks using the SSICA algorithm, and let the algorithm extract up to three specific networks per condition. Corrections for multiple comparisons at the cluster level were performed using Gaussian random field theory as implemented in FSL software (minimum $z>3$; cluster significance, $p<0.05$, corrected).

We then used the $z$-score spatial map of each extracted specific component as regressor in a general linear model to estimate the contribution of the specific network over time in each subject and each run (Fig. 2C). In contrast to the regular application of GLM in fMRI, where a spatial map is estimated from a time series, here a time course is estimated based on the relative weight of each voxel in the spatial map. This time course measures the integrity of the specific network (or the strength of functional connectivity within different voxels of this network) at each time point in different resting-state runs of each subject and can be used as a measure of within-network functional integration. We acquired two runs of resting state in each of the pretraining and post-training conditions. The first and the second resting-state runs in the post-training condition were acquired $\sim 30$ and $55 \mathrm{~min}$ following the end of perceptual training, respectively. We investigated dynamic changes in the strength of functional connectivity within the specific network over the course of the two runs of the pretraining condition and the two runs of the posttraining condition. We used short-time Fourier transform (STFT; with the standard Hamming window as implemented in MATLAB) to assess changes in power over the estimated time course, described above, within the neural activity-related frequency band of the resting-state BOLD signal (0.009-0.08 Hz; Fox and Raichle, 2007). Two-factor ANOVA with repeated measures was used to perform group-level statistics on the average power calculated from each run of each subject (factors: time and training condition). Figure $2 C$ summarizes the computational pipeline used in this procedure.

\section{Results}

We studied the short-term neural correlates of perceptual learning (within $1 \mathrm{~h}$ ) by analyzing changes in the functional connectivity during resting-state periods following perceptual somatosensory training. In the perceptual training, which was performed outside the scanner, a robotic device passively moved the arm, which was hidden from view, outward along one of a set of fanshaped paths (for more details, see Materials and Methods). Subjects were required to judge whether the robot displaced the hand to the right or the left of the midline, and feedback on response accuracy was provided. The experimental design is illustrated in Figure $1 A$. To assess persistent changes in both the brain and motor behavior, which are associated with perceptual training, the training was preceded and followed by resting-state fMRI scanning sessions as well as null-field reaching movements. A force-field motor learning task followed the second set of null-field movements to measure mo- 
tor learning following somatosensory training in a learning paradigm that uses joint movements that are similar to those used during the perceptual training.

We obtained quantitative measures of perceptual change for subjects in the somatosensory discrimination task. The perceptual index (Eq. 4) decreased over the course of training, which indicates improvements in perceptual classification accuracy (Fig. $1 B ; t_{(13)}=5.2 ; p<0.0002$, the first vs the last block of training). We observed that, with training, the perceptual boundary approached the actual boundary between left and right, and perceptual acuity increased (Fig. $1 C$; $p<$ 0.001 in both cases). To rule out the possibility of active motor outflow during perceptual training, we examined the forces that subjects exerted on the robot handle during training. Measured forces were low throughout, averaging $0.52 \mathrm{~N}$ $( \pm 0.20)$ orthogonal to the displacement and $0.68 \mathrm{~N}( \pm 0.23)$ in line with the displacement. The measured forces did not vary in any systematic fashion over the course of training or with the movement direction.

During active reaching movements, the subject was required to move straight from the start to the end positions. We assessed the effects of perceptual training on motor control and learning by measuring the curvature of the hand path (maximum lateral deviation of the hand from a straight-line path; PD) on a trial-by-trial basis. Figure $1 A$ shows $\mathrm{PD}$ values averaged across subjects during null-field and forcefield trials. It can be seen that lateral deflections are significantly reduced in the post-training null-field movements compared with the pretraining baseline values $(p=0.02$, average PD over the last 50 trials of pretraining vs post-training null-field conditions). The straighter hand trajectories during active movements following somatosensory training reflect the influence of perceptual training on the motor domain and motor control. Figure $1 A$ also shows that the force-field initially resulted in a substantial lateral deviation, which was reduced over the course of motor learning. It is seen that lateral deviation is eliminated as early as trial 5, but asymptotic performance is not achieved until trial 25 or 30 .

To better quantify the degree of motor learning, we measured the amount of lateral force applied during channel trials early (trial 15), midway (trial 85), and late (averaged over trials 135, 139 , and 143) in learning. Figure $1 D$ shows measured force profiles in blue averaged across subjects. The red curves in Figure $1 D$ show the ideal force profile that subjects would need to exert to fully compensate for the force-field perturbation. The ideal force profiles were not statistically different in terms of peak force $\left(F_{(2,26)}=2.4, p>0.1\right)$ or area under the curve $\left(F_{(2,26)}=1.5, p>\right.$ $0.2)$. As shown, subjects progressively learned to apply force in an accurate spatial and temporal pattern to compensate for the newly changed dynamics of the environment. We also examined the relationship between the amounts of perceptual learning (change in the perceptual index over the course of somatosensory training) and motor learning as measured by the force index (Eq.
3). We found that those subjects who learned more during perceptual training, subsequently exhibited greater amounts of motor learning $(r=0.64, p=0.01$; Fig. $1 E)$. A further analysis was performed of the relationship between motor learning (force index) and changes in perceptual performance due to perceptual training as measured by the slope and intercept of the fitted psychometric function. We found no relationship between motor learning and changes in the intercept (bias, $p>0.3$ ). In contrast, motor learning was marginally correlated with improvements in perceptual acuity $(r=0.50, p=0.06)$.

As described earlier, we acquired resting-state fMRI scans that were interleaved with the perceptual training. Resting-state scans were conducted with the eyes closed. There was no explicit task. By scanning under resting-state conditions, any changes that we observe in functional connectivity between sessions can be attributed to plasticity induced by perceptual learning rather than to pretraining versus postlearning differences in the performance of the behavioral task or attentional differences during task performance before and after learning. To identify those changes in the brain functional connectivity that are related to behavioral improvements (as opposed to nonspecific changes that may be observed between the two resting-state conditions), various behavioral indices were used as a regressor in the seed-based functional connectivity analyses, as described in the Materials and Methods. In general, the motor control index, measuring improvement in the performance accuracy of null-field movements following perceptual training (Eq. 2, MI), accounted for more of the variability in functional connectivity changes than the other 


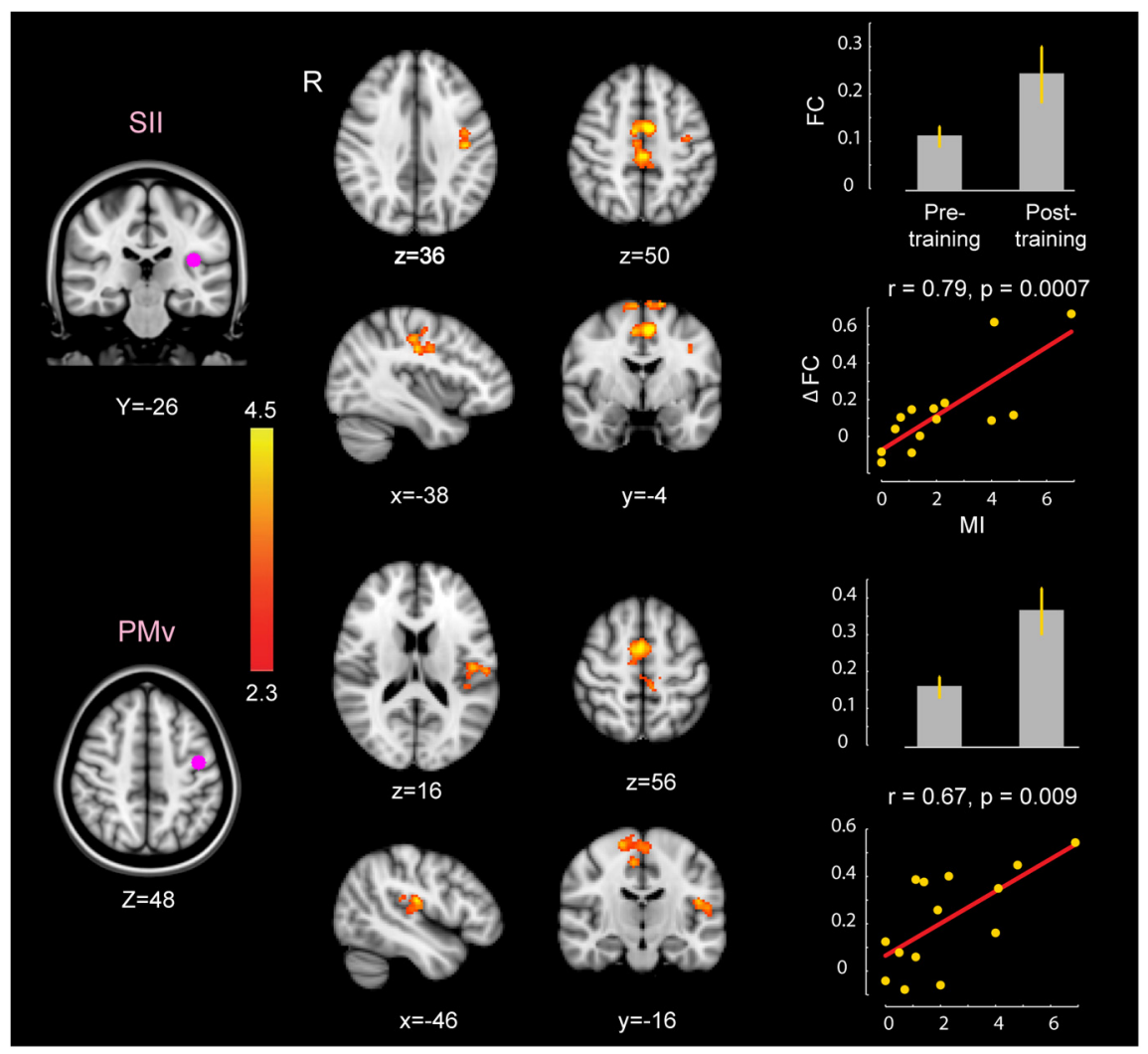

Figure 4. Changes in partial correlation of seed regions at the second level of the perceptual processing sequence (SII and PMv) in relation to motor performance change. In the partial correlation analysis, the effects of signals recorded from the primary somatosensory areas are removed. Display conventions are as in Figure 3.

behavioral factors such as PI (changes in perceptual accuracy; Eq. 4) or FI (degree of force-field learning; Eq. 3). Therefore, we first report on the results of analysis using $\mathrm{MI}$ as a regressor and then illustrate those with respect to PI and FI.

We first tested the hypothesis that perceptual training not only changes sensory areas of the brain, but also effects changes within the motor circuit that cannot be explained by changes in the somatosensory system. Figures 3, 4, and 5 show, in a sequential fashion (Fig. 2B, perceptual processing model), those changes in functional connectivity that are strengthened as a result of perceptual learning and are specifically related to the post-training improvements in motor performance (MI as the regressor). Figure 3 shows training-specific changes in the functional connectivity of the ROIs at the first level of perceptual processing hierarchy, which includes different parts of primary somatosensory cortex. Each row shows seed regions at the left and, to the right, those clusters whose correlation with the seed region increases reliably as a result of perceptual training. In each case, initially positive correlations are increased following training. Subjects who experience a greater change in the motor index show greater changes in connectivity in the resting brain. In particular (Fig. 3, top), somatosensory training increases functional connectivity between BA2 and bilateral primary somatosensory cortex (SI) (BA1/2), left M1, PMd, and the superior parietal lobule (Table 2). Figure 3 (bottom) shows strengthening of the connections, as a result of somatosensory training, between BA3b and bilateral primary motor, and dorsal premotor cortices and right BA1/2. Also, as shown in Figure 6 top row, similar results are observed using PI as an explanatory variable in a general linear model contrasting post-training versus pretraining conditions.
Overall, these analyses show that the elevation of functional connectivity in a network composed of bilateral anterior parietal and dorsal frontal motor areas is tightly related to the behavioral improvements in both motor and somatosensory perceptual domains.

Figure 4 shows the effects of somatosensory training on ROIs in the second level of the perceptual processing hierarchy (Fig. 2B), after removal of the BOLD signal correlates of primary somatosensory cortex. Figure 4 demonstrates trainingdependent changes in functional connectivity between SII (top row) and PMv (bottom row), and the rest of the brain that cannot be explained by activity in primary somatosensory cortex. The functional connectivity between SII and bilateral M1 and SMA, and left PMv is increased by the effects of somatosensory training. Somatosensory training is also seen to strengthen the connection between PMv as ROI and two clusters, one located in the left SII and the other one in bilateral SMA and M1 (Table 2). Note that the changes in connectivity in the network composed of SII, PMv, SMA, and M1 are independent of activity in primary somatosensory areas. The change in functional connectivity of this network following perceptual training is tightly correlated with the behavioral improvements in motor performance (MI measure). Using PI as the regressor, there is no reliable change in connectivity related to SII and PMv, when the activity in SI is regressed out.

The above analyses treat SII and PMv at the same level of the model shown in Figure $2 B$. In support of this decision, we tried to separately regress out the effects of SI and SII activation from PMv. We found that after doing so there were no reliable learning-related changes in connectivity between PMv and other areas of the brain. We also tried to regress out the effects of SI and PMv from SII, which likewise left no reliable changes in connectivity between SII and other brain areas. SII and PMv thus appear to have similar functions in the perceptual decisionmaking sequence.

To investigate whether the changes in frontal motor areas following perceptual training are a byproduct of changes in somatosensory areas or whether perceptual training results in independent changes in the motor system, we partialled out activity from the first and second levels of the perceptual processing stream while examining changes in the frontal motor areas. Figure 5 (top) shows training-dependent changes in functional connectivity with a seed in SMA, when activity attributable to SI, SII, and PMv is removed. One observes an increase in connectivity between SMA and clusters in bilateral dorsal primary and premotor cortices, as well as with right cerebellar cortex Crus I and lobule VI (Table 2). The absolute value of the correlation is increased by the effects of training in both clusters (an increase in positive correlation with the cortical cluster and an increase in negative correlation with the cerebellar cluster). The increase in negative correlation between the frontal motor areas and the cerebellar cortex is consistent with previous reports of resting- 
state and task-based networks following motor learning (Penhune and Doyon, 2005; Ma et al., 2010; Vahdat et al., 2011), and also with the inhibitory influence of cerebellar cortex on the frontal motor areas of the brain. Somatosensory training likewise strengthens the connection between the left and right M1, when the effects of all the somatosensory and nonprimary motor areas were removed (Fig. 5, bottom row). Of note, the modifications in connectivity within frontal motor areas following somatosensory training are specifically correlated with behavioral measures of how accurately subjects move their limb (MI), but not how accurately they perceive their limb movements (PI).

There are also brain areas that show a decrease in the strength of connectivity following perceptual training. Specifically, PI was the only behavioral measure that was correlated with a decrease in connectivity post-training. Figure 6 (middle and bottom rows) shows those areas whose functional connectivity with primary sensorimotor seed regions decreased following perceptual training. These areas, color coded in blue, include posterior cingulate cortex and precuneus, which are parts of the so-called default mode network (Buckner et al., 2008). In each case, it can be seen that an initially negative correlation approaches zero following training (Table 3). Thus, it is seen that perceptual learning serves to disengage primary sensorimotor areas of the resting brain from the posterior parts of the default mode network. Notably, the disengagement is observed at an average time of $\sim 1 \mathrm{~h}$ following sensory training when the resting-state scans were conducted. Consolidation of perceptual learning is presumably dependent on the separation of specific sensorimotor pathways from the noninvolved default-mode areas of the brain.

We also investigated whether changes in functional connectivity following perceptual training can predict the subsequent degree of force-field motor learning. Figure 7 shows those changes in functional connectivity that are correlated with the FI measure (force compensation at the end of learning) using the partial functional connectivity analysis described earlier. Left M1 was the only seed region that showed reliable post-training change in connectivity correlated with FI. Two clusters were identified; one cluster comprised bilateral primary motor cortices whose connectivity significantly increased following perceptual training in relation to the degree of force-field learning $(r=0.69, p=0.006$; Fig. 6, top row). A second cluster was located in the left putamen, which showed a significant change in correlation, from positive to negative values, with the M1 seed following training (Table 4). The magnitude of changes in functional connectivity was marginally correlated with the FI measure $(r=0.50, p=0.06$; Fig. 6 , bottom row).

We assessed the possibility that the changes we have observed in cortical motor areas following perceptual training might be related to activity in sensorimotor areas that we have not included in the hierarchical model shown in Figure $2 B$. In particular, we tested for the effects of seeds in the left sensorimotor thalamus (MNI coordinates: $x=-16, y=-26, z=6)$, left putamen $(-26$, $-8,2)$, left superior parietal lobule (BA7; $-10,-78,48)$, and right cerebellar cortex $(24,-46,-26)$, after removal of the BOLD signal correlates of primary somatosensory cortex. The seed regions for these tests were obtained from the peak of activity in that area during localizer scans that were run at the end of the scanning sequence. None of these areas showed changes in connectivity that were correlated with any of the behavioral measures used in this study. Thus, changes in the sensorimotor network following perceptual learning are primarily associated with those areas shown in Figure $2 B$.

To investigate the temporal pattern of the off-line changes that occur within the hour following the end of perceptual training, we used a data-driven approach called shared and specific independent component analysis (Vahdat et al., 2012) in combination with temporal GLM and STFT analysis, as described in the Materials and Methods (Fig. 2C). This method allows the extraction and classification of brain networks that are significantly more activated during pretraining or post-training conditions (called specific networks). This analysis resulted in two networks that were specific to the post-training scans, that is, following perceptual training. There were no networks that were specific to the pretraining scans alone (Fig. $8 A, D$ ). The first specific network, associated with perceptual training, includes left PMd, M1, SI, and the posterior parietal cortex (Fig. 8A; Table 5). The strength of functional connectivity within this network, as assessed by the average power, is significantly increased from pretraining to post-training resting-state conditions $\left(F_{(1,13)}=39.3\right.$, 
Table 2. Summary of results using the MI as a regressor to predict functional connectivity

\begin{tabular}{|c|c|c|c|c|c|c|c|c|}
\hline \multirow[b]{2}{*}{ ROI } & \multirow[b]{2}{*}{$P_{\text {corr }}$} & \multirow[b]{2}{*}{$z$-value } & \multicolumn{3}{|c|}{ MNI coordinates } & \multicolumn{2}{|l|}{$z$-value } & \multirow[b]{2}{*}{ Anatomical label } \\
\hline & & & $x$ & $y$ & $z$ & Pretraining & post-training & \\
\hline \multirow[t]{4}{*}{ BA2 } & 0.0000 & 4.5 & -48 & -40 & 54 & 3.3 & 5.1 & L anterior intraparietal sulcus \\
\hline & & 4.2 & -60 & -12 & 40 & 4.2 & 6.3 & L postcentral gyrus, BA1 \\
\hline & 0.02 & 4.2 & -42 & -8 & 60 & 3.0 & 5.0 & L precentral gyrus, BA6 \\
\hline & & 4.3 & 50 & -26 & 44 & 3.7 & 5.7 & R postcentral gyrus, BA2 \\
\hline \multirow[t]{3}{*}{ BA3b } & 0.0000 & 5.6 & 6 & -26 & 76 & 4.7 & 7.1 & L precentral gyrus, BA4 \\
\hline & & 4.5 & -2 & -28 & 68 & 5.3 & 7.4 & R precentral gyrus, BA4 \\
\hline & & 5.0 & 24 & -40 & 68 & 8.8 & 9.9 & R postcentral gyrus, BA1/2 \\
\hline \multirow[t]{4}{*}{ SII } & 0.0002 & 4.9 & -6 & -4 & 50 & 0.9 & 4.0 & SMA, BA6 \\
\hline & 0.0003 & 4.5 & 12 & -24 & 76 & 2.0 & 3.4 & R precentral gyrus, BA4 \\
\hline & 0.02 & 4.1 & -40 & -20 & 42 & 1.3 & 4.3 & L precentral gyrus, BA4 \\
\hline & & 3.2 & -60 & -4 & 30 & -1.0 & 2.2 & LPMv, BA6 \\
\hline \multirow[t]{3}{*}{ PMv } & 0.0000 & 4.4 & 2 & 0 & 56 & 4.4 & 7.2 & SMA, BA6 \\
\hline & 0.007 & 3.9 & 18 & -16 & 66 & 1.7 & 4.9 & R precentral gyrus, BA6 \\
\hline & & 4.4 & -46 & -18 & 18 & 0.3 & 3.7 & LSII, parietal operculum OP1 \\
\hline \multirow[t]{5}{*}{ SMA } & 0.0000 & 4.3 & 12 & -30 & 62 & 3.4 & 5.8 & R precentral gyrus, BA4 \\
\hline & & 4.0 & 38 & -12 & 68 & 0.1 & 3.2 & R precentral gyrus, BA6 \\
\hline & 0.0001 & 4.0 & -14 & -14 & 62 & 5.6 & 8.3 & L precentral gyrus, BA6 \\
\hline & & 5.0 & 26 & -58 & -24 & 0.9 & -3.1 & R Cerebellum, Crus I \\
\hline & & 3.3 & 28 & -72 & -24 & 1.2 & -2.7 & R Cerebellum, lobule VI \\
\hline \multirow[t]{3}{*}{ M1 } & 0.002 & 4.3 & 18 & -30 & 60 & 3.9 & 6.9 & R precentral gyrus, BA4 \\
\hline & & 4.1 & 10 & -24 & 74 & 6.7 & 8.1 & R precentral gyrus, BA6 \\
\hline & & 4.0 & -6 & -28 & 60 & 5.4 & 7.5 & L precentral gyrus, BA4 \\
\hline
\end{tabular}

The table shows clusters whose correlation with the corresponding ROI increased reliably with perceptual training. $P_{\text {corr }}$ Corrected cluster-level $p$ value related to the regressor of interest (MI); R, right; L, left. The associated $z$-score for the peak is shown in the $z$-value column. $z$-values pertaining and post-training give $z$-scores at the location of maximum activation based on values averaged over subjects in pretraining and post-training conditions respectively.

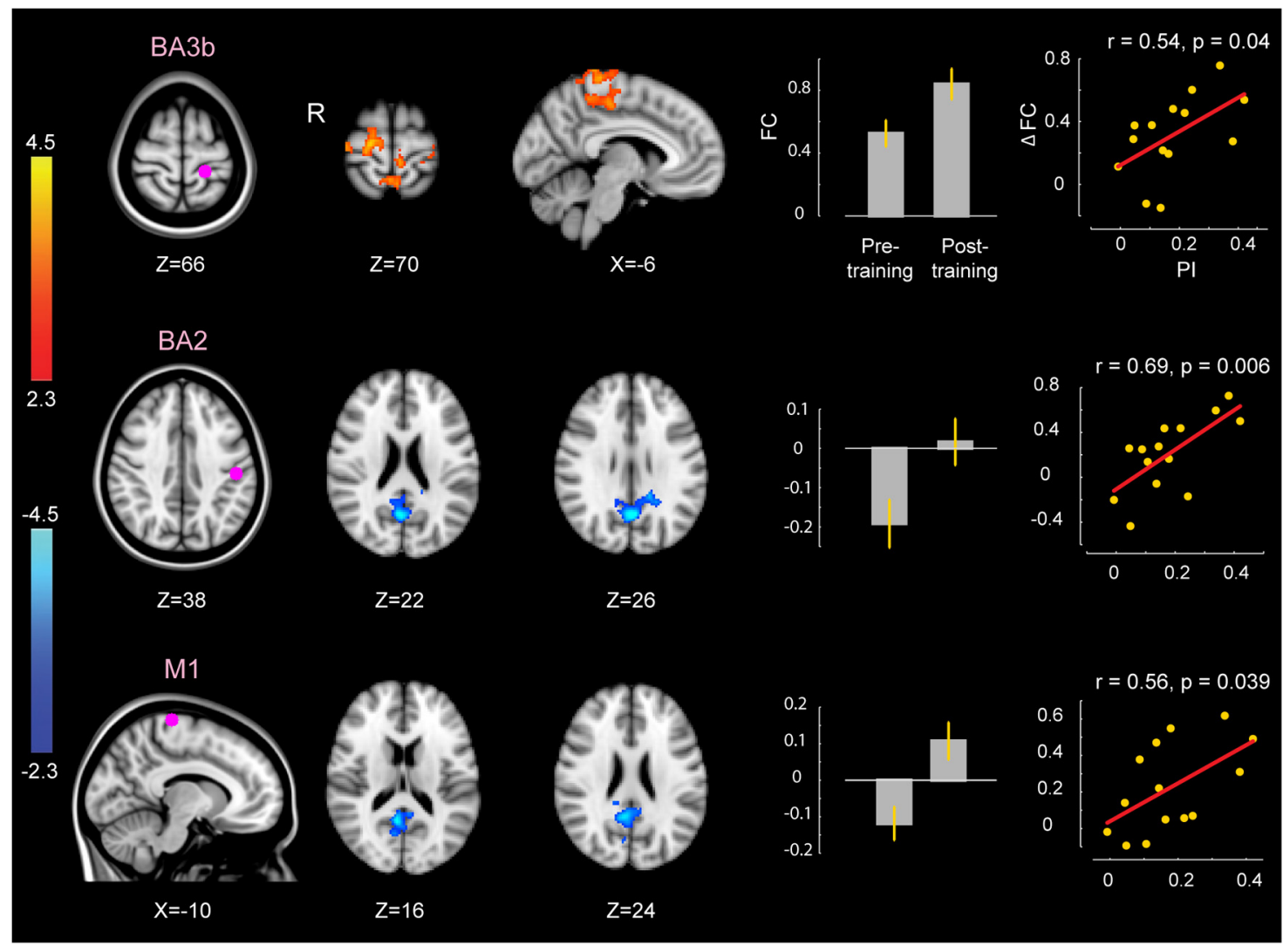

Figure 6. Changes in connectivity in relation to perceptual change. Perceptual learning disengages primary sensorimotor regions from posterior parts of the default mode network during resting-state periods following the completion of training. Red and blue color bars indicate, respectively, increase and decrease in the magnitude of connectivity following training. Display conventions are as in Figure 3.

$p<0.00005)$. The second specific network corresponding to post-training includes bilateral SII, SI, and PMv areas (Fig. 8D; Table 5) also increases in power in post-training scans $\left(F_{(1,13)}=\right.$ $11.7, p<0.005)$.
Having acquired two resting-state fMRI scans per condition allowed us to further examine how the power of the specific networks changes over the hour following the end of training. Figure $8, B$ and $E$, gives the temporal profile of the power of these specific 
Table 3. Summary of results using the PI as a regresssor to predict functional connectivity

\begin{tabular}{|c|c|c|c|c|c|c|c|c|}
\hline \multirow[b]{2}{*}{ ROI } & \multirow[b]{2}{*}{$P_{\text {corr }}$} & \multirow[b]{2}{*}{$z$-value } & \multicolumn{3}{|c|}{ MNI coordinates } & \multicolumn{2}{|l|}{ z-value } & \multirow[b]{2}{*}{ Anatomical label } \\
\hline & & & $x$ & $y$ & $z$ & Pretraining & Post-training & \\
\hline \multirow[t]{4}{*}{ BA3b } & 0.0000 & 4.6 & -2 & -22 & 46 & 6.6 & 8.8 & L primary motor cortex, BA4 \\
\hline & 0.006 & 3.9 & -12 & -20 & 62 & 5.8 & 7.5 & L precentral gyrus, BA6 \\
\hline & 0.001 & 3.6 & -4 & -52 & 68 & -0.1 & 3.5 & L superior parietal lobule, BA7 \\
\hline & & 4.1 & 18 & -18 & 70 & 6.3 & 7.9 & R precentral gyrus, BA6 \\
\hline BA2 & 0.0000 & 4.7 & 0 & -62 & 30 & -4.3 & -0.6 & Precuneus cortex \\
\hline \multirow[t]{2}{*}{ M1 } & 0.0001 & 4.7 & 8 & -54 & 20 & -3.1 & 1.8 & Precuneus cortex \\
\hline & & 3.5 & -6 & -48 & 14 & -2.3 & 1.0 & Posterior cingulate gyrus \\
\hline
\end{tabular}

Details are as in Table 2.

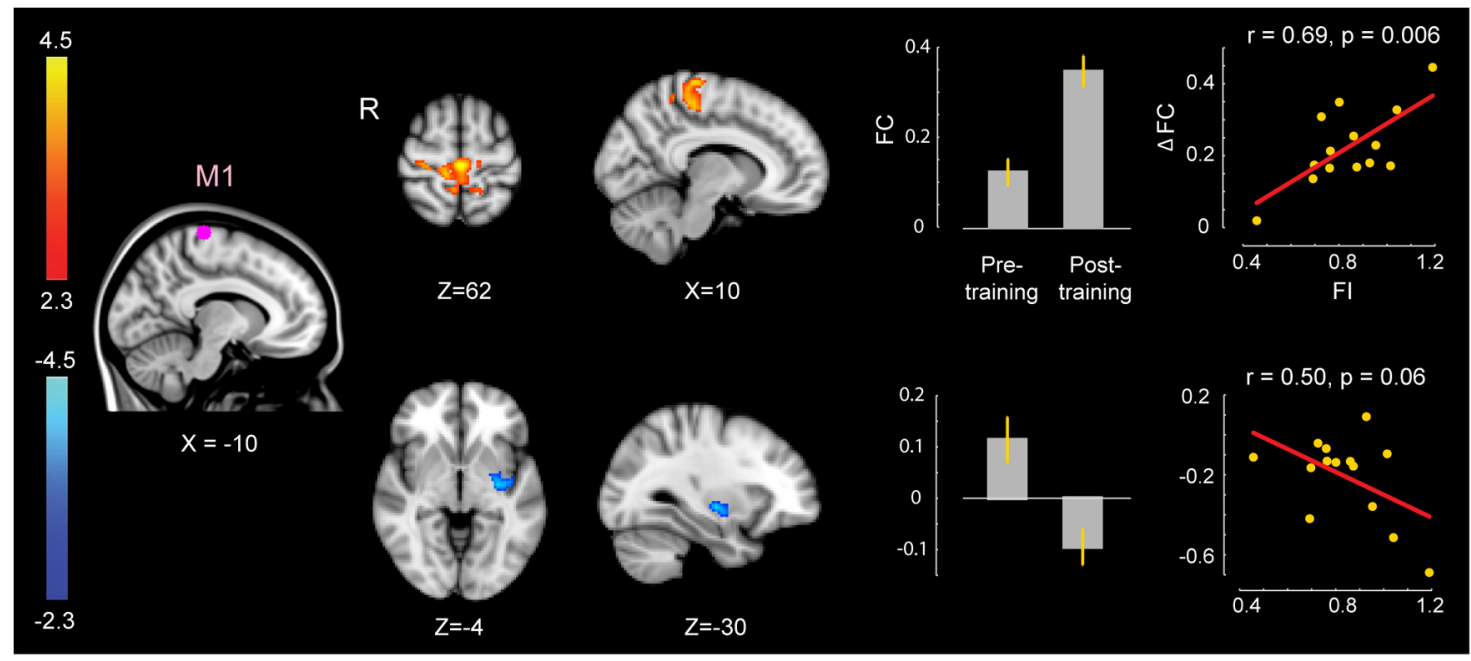

Figure 7. Changes in connectivity as a result of perceptual learning in relation to the degree of subsequent motor learning. M1 is the only seed region that showed a significant change in functional connectivity in association with the FI measure. This figure shows the result of partial correlation analysis after the removal of all the previous areas in the perceptual-processing sequence. Display conventions are as in Figure 3.

Table 4. Summary of results using the $\mathrm{Fl}$ as a regresssor to predict functional connectivity

\begin{tabular}{|c|c|c|c|c|c|c|c|c|}
\hline \multirow[b]{2}{*}{ ROI } & \multirow[b]{2}{*}{$P_{\text {corr }}$} & \multirow[b]{2}{*}{$z$-value } & \multicolumn{3}{|c|}{ MNI coordinates } & \multicolumn{2}{|l|}{$z$-value } & \multirow[b]{2}{*}{ Anatomical label } \\
\hline & & & $x$ & $y$ & $z$ & Pretraining & Post-training & \\
\hline M1 & $\begin{array}{l}0.0000 \\
0.009\end{array}$ & $\begin{array}{l}4.8 \\
4.2 \\
4.2\end{array}$ & $\begin{array}{r}-2 \\
10 \\
-30\end{array}$ & $\begin{array}{l}-26 \\
-28 \\
-12\end{array}$ & $\begin{array}{r}62 \\
72 \\
-4\end{array}$ & $\begin{array}{l}5.0 \\
7.1 \\
2.8\end{array}$ & $\begin{array}{r}7.4 \\
8.6 \\
-2.3\end{array}$ & $\begin{array}{l}\text { L precentral gyrus, BA4a } \\
\text { R precentral gyrus, BA4a } \\
\text { L putamen }\end{array}$ \\
\hline
\end{tabular}

Details are as in Table 2

networks at different time points averaged over subjects (see Materials and Methods). As shown, the power of both networks (which represent the strength of functional connectivity within each network) is consistently higher in the post-training restingstate runs (blue curves) compared with the pretraining runs (red curves). An ANOVA, applied to the first specific network comprising dorsal frontal motor and parietal areas, showed that the change in power between the first and second scanning runs, was dependent on whether it was measured before or after the perceptual training (significant interaction, $F_{(1,13)}=4.6, p=0.05$; Fig. $8 C$ ). Post hoc analysis revealed significantly more power in this network during the second run of post-training compared with the first run $\left(t_{(\mathrm{df}=13)}=3.5, p<0.005\right)$. Of note is the observation that the power in this network peaks at $\sim 50 \mathrm{~min}$ following the end of perceptual training, suggesting ongoing neural processing during this period. There was no reliable change between the two pretraining runs ( $p>0.2$; Fig. $8 C$, red curves). A second ANOVA on the second specific network found no reliable interaction between the two scanning runs and pretraining versus post-training conditions ( $p>0.3$; Fig. $8 F$, blue curves).

\section{Discussion}

The present findings show that perceptual training induces plasticity in the human motor system that cannot be explained by activity in the somatosensory network. Perceptual training is seen to change the characteristics of subsequent movements and to improve somatosensory perceptual judgments. We have used a neural model of perceptual processing in conjunction with a partial correlation technique to parcel out changes in connectivity associated with each of the levels of this model. Changes in brain networks associated with perceptual training are seen at all stages of the somatosensory processing hierarchy. At each step of the processing sequence, plasticity is linked to different behavioral outcomes. When the bottom-up effects of somatosensory inputs are removed, there still remain changes in connectivity in frontal motor areas that are linked to perceptual learning.

Overall, we have observed that perceptual learning results in changes in functional connectivity between primary somatosensory cortex and frontal motor areas (M1 and PMd bilaterally). When the effects of activity in SI were removed, we found that 

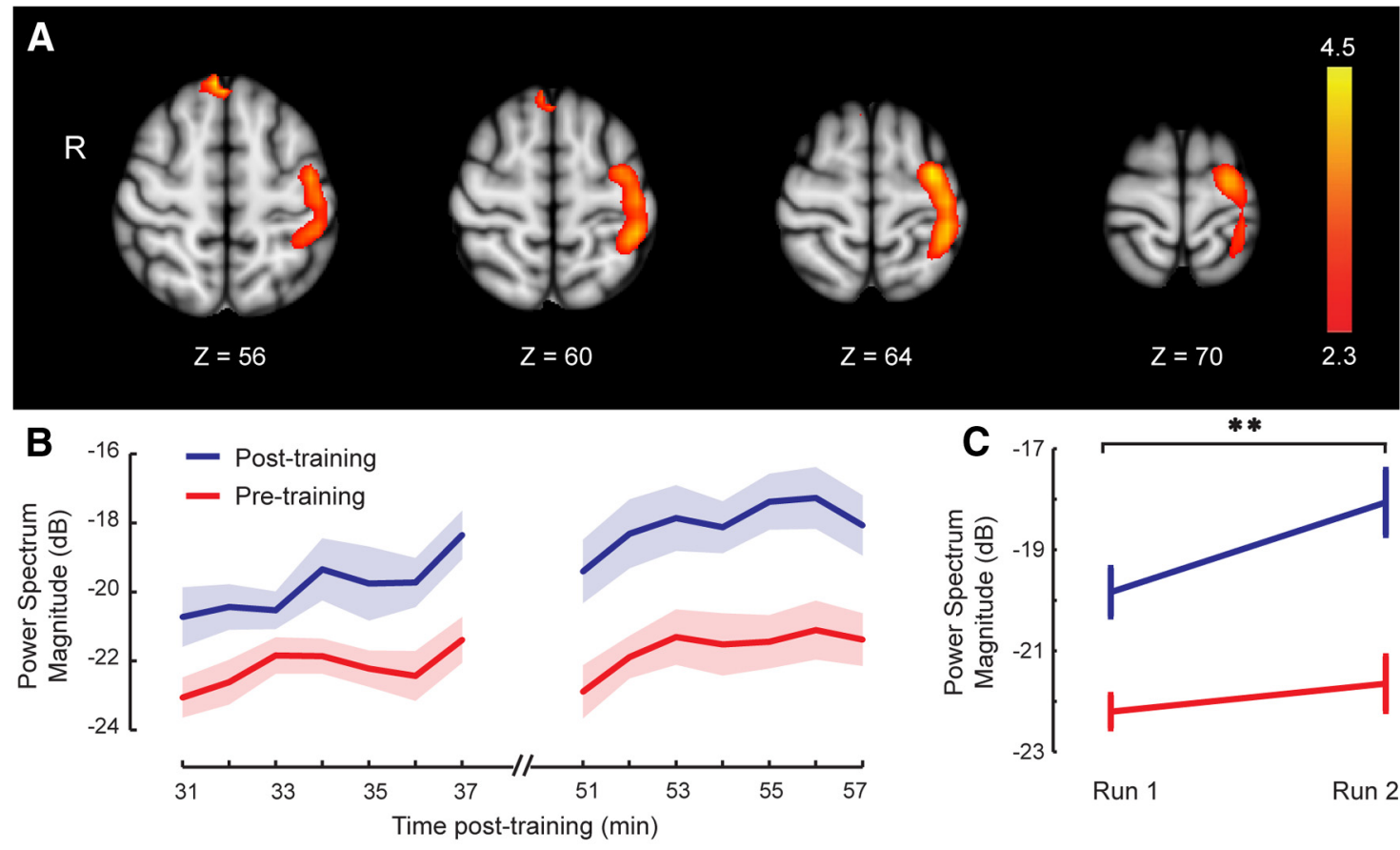

Run $1 \quad$ Run 2
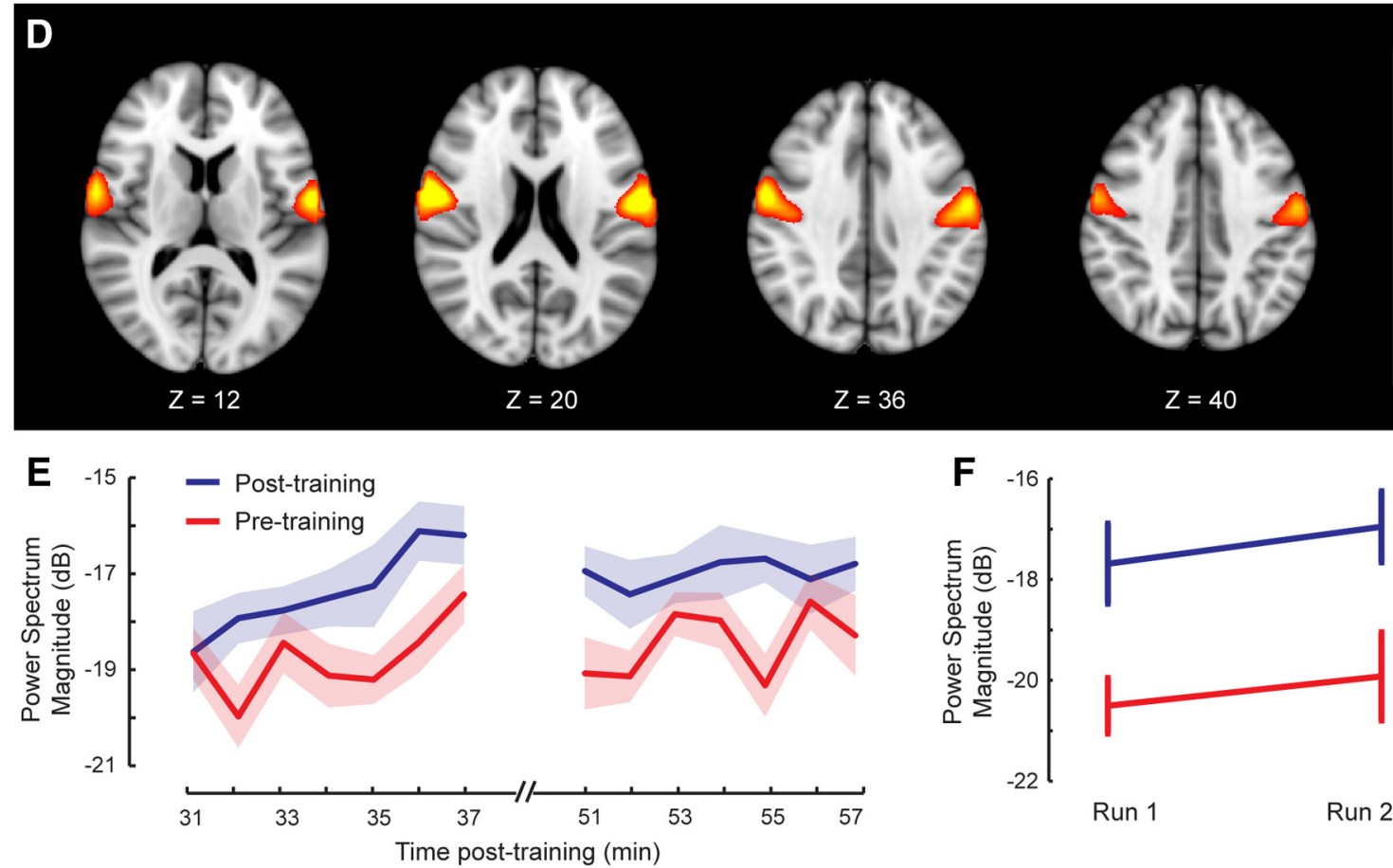

Run 1

Run 2

Figure 8. Resting-state networks specific to the postlearning scans and their temporal power profile before and after perceptual learning. $\boldsymbol{A}$ and $\boldsymbol{D}$ show $\boldsymbol{Z}$-score spatial maps of two specific components corresponding to the postlearning condition, using the SSICA analysis. $\boldsymbol{B}$ and $\boldsymbol{E}$ show the temporal profile of the power of networks shown in $\boldsymbol{A}$ and $\boldsymbol{D}$ at different time points averaged over subjects before (red) and after (blue) perceptual learning. $\boldsymbol{C}$ and $\boldsymbol{F}$ show the mean power of networks shown in $\boldsymbol{A}$ and $\boldsymbol{D}$ in each of the two resting-state runs performed before (red) and after (blue) perceptual learning averaged over subjects. Power magnitudes are given in decibels. Shaded area represents the SEM.

seeds placed separately in SII and PMv resulted in reliable changes in connectivity with SMA and M1, and with one another. When the contributions of activity in SI, SII, and PMv were also removed, there still remained reliable changes in connectivity related to perceptual learning in the links between SMA and M1, PMd and cerebellar cortex, and also bilaterally in M1. In effect, we see that there are distinct perceptual learning-related changes in connectivity at all of the modeled stages of the somatosensory decision-making sequence. In motor areas, in particular, there are changes to connectivity patterns that are related to activity in the somatosensory network, and, additionally, there are also changes that are uncorrelated with somatosensory activity. This may reflect different mechanisms underlying plasticity in motor areas following perceptual training, as suggested by correlations with behavioral measures that were related to sensory (PI) versus motor learning (FI) variables.

Much of what we know about motor learning has come from studies that have examined how subjects adapt to altered sensory 


\begin{tabular}{|c|c|c|c|c|c|c|}
\hline \multirow{2}{*}{$\begin{array}{l}\text { Network } \\
\text { index }\end{array}$} & \multirow{2}{*}{$\begin{array}{l}p \text { value } \\
\text { (post - pre) }\end{array}$} & \multirow[b]{2}{*}{ z-value } & \multicolumn{3}{|c|}{ MNI coordinates } & \multirow[b]{2}{*}{ Anatomical label } \\
\hline & & & $x$ & $y$ & $Z$ & \\
\hline \multirow[t]{4}{*}{1} & \multirow[t]{4}{*}{0.0000} & 4.9 & -26 & -10 & 68 & L precentral gyrus, BA6 \\
\hline & & 4.4 & -42 & -38 & 60 & L postcentral gyrus, BA2 \\
\hline & & 4.0 & -40 & -22 & 60 & L precentral gyrus, BA4 \\
\hline & & 3.3 & -26 & -50 & 68 & L superior parietal lobule, BA7 \\
\hline \multirow[t]{4}{*}{2} & \multirow[t]{4}{*}{0.004} & 9.7 & -58 & -6 & 24 & L postcentral gyrus, BA3b \\
\hline & & 8.3 & -60 & -6 & 12 & L SII, parietal operculum OP4 \\
\hline & & 8.1 & -58 & -4 & 30 & LPMv, BA6 \\
\hline & & 7.3 & -52 & -8 & 34 & L precentral gyrus, BA4 \\
\hline
\end{tabular}

$p$ values (post - pre), the result of statistical tests comparing the strength of functional connectivity within each network from pretraining to post-training resting-state scans; $L$, left. $z$-values give the $z$-scores of the activation peaks in the spatial map of each specific network.

feedback. Typically, visual or somatosensory information is altered as subjects make movements to generally well defined sensory targets. But outside of the laboratory, the sensory targets of movements, and in particular those associated with somatic function, are themselves inexact and must be refined with learning and practice. In the present study, we have intentionally separated perceptual and motor learning to better understand the somatosensory changes that accompany motor learning and to determine the extent to which changes in motor areas of the brain are produced by perceptual learning alone.

Our behavioral data indicate that somatosensory judgments are rapidly modified with feedback, that perceptual acuity increases with training, and that movements are rapidly realigned to reflect this perceptual learning. In our neuroimaging analyses, we have based the identification of networks on a model of the perceptual decision-making process that is derived from work on nonhuman primates. We are thus able to identify functional connections that are not linearly related to activity earlier in the sequence. Without this kind of correction, it would be difficult to determine whether or not the activations in a given area (or connectivity between areas) can be explained by patterns elsewhere in the brain or whether they might have a distinct functional role. While our analysis does not preclude the possibility of a nonlinear dependence of motor areas on activity in sensory regions, it does eliminate the simplest version of this idea, that motor areas are simply mirroring the activity in sensory regions. The results of this analysis lend support to the idea that perceptual learning is actually changing motor areas of the brain. However, it should be noted that the present analyses were conducted on the basis of low-frequency BOLD signal fluctuations under resting-state conditions. This does not rule out the possibility of higher-frequency dependencies in electrophysiological activity patterns in the sensorimotor network.

The connectivity analyses reported in the present article are tied to behavioral measures of learning. That is, the connectivity analyses identify correlations in the hemodynamic response that are directly related to the changes in movement following perceptual learning. By linking the connectivity analyses to behavioral measures of learning, we rule out the possibility that any observed effects are due to nonspecific changes that occur between the two resting-state scans. In the present analyses, changes in connectivity that are associated with earlier stages of the sensory-processing model are related to both motor and perceptual variables (MI and PI). At later stages, changes to connectivity measures are linked only to improvements in motor performance and learning (MI and FI). This suggests that changes in the voluntary control of movement that are associated with perceptual training arise from a distributed pattern of plasticity throughout the entire sensorimotor network.

Aspects of the time course of changes to the sensorimotor network were obtained by using ICA to assess the strength of networks over the course of the pretraining and post-training scans. Using a technique that extracts components that are shared between pretraining and post-training scans and those that are specific to each (Vahdat et al., 2012), we identified two networks that are specific to the post-training scan, one consisting of SII and PMv, and the other of left sensorimotor cortex, BA7, and PMd. The latter network showed a progressive increase in power over the course of the scanning sequence. Of note is the observation that there is a local maximum for power at $\sim 50 \mathrm{~min}$ following the end of perceptual training, suggesting ongoing neural processing during this period.

Some differences between the ICA and seed-based analyses should be noted. In particular, whereas the seed-based analyses detected changes in connectivity that were related to behavioral measures, ICA identified changes in connectivity from pretraining to post-training regardless of behavioral factors. Moreover, in the seed-based analyses, but not ICA, a partial correlation procedure was used to account for activity in somatosensory areas. Nevertheless, the two techniques resulted in comparable networks. In particular, the networks reported in Figures 3 (BA2) and 4 match those obtained in Figures $8, A$ and $D$, respectively.

The present findings complement those of similarly constructed behavioral studies that assess the effects of perceptual training on force-field adaptation (Darainy et al., 2013). In the Darainy et al., 2013 study, we tested different aspects of perceptual training and its effect on motor performance, perception, and learning. It was found that, compared with control subjects that received no perceptual training, a protocol identical to that used here resulted in increases in the rate and the extent of motor learning regardless of whether the shift in the perceptual boundary served to increase or decrease the magnitude of sensory error. Somatosensory perceptual acuity was the primary variable associated with improvements in motor learning following training. Thus, as in the present study, these findings suggest that changes to movement during sensorimotor adaptation may in part be the result of perceptual learning.

A number of previous studies have examined the effects of somatosensory stimulation on human motor function. Several studies have assessed brain activity following somatosensory training using passive movement of the upper limb (Carel et al., 2000; Kaelin-Lang et al., 2005; Macé et al., 2008). Some studies have observed no changes in motor performance or motor areas of the brain, or no change in corticomotor neural excitability following periods of passive training (Lotze et al., 2003; KaelinLang et al., 2005). Others have documented changes to sensorimotor areas (Pleger et al., 2003), and changes in corticospinal excitability in both healthy (Carel et al., 2000; Lewis and Byblow, 2004; Macé et al., 2008) and patient populations (Nelles et al., 2001; Lindberg et al., 2004; Dechaumont-Palacin et al., 2008). Other forms of somatosensory stimulation, such as peripheral nerve stimulation (Conforto et al., 2002; Charlton et al., 2003), muscle tendon vibration (Forner-Cordero et al., 2008), and paired associative stimulation (Stefan et al., 2000; Stefan et al., 2002), have resulted in enhanced corticospinal excitability and changes in corticomotor representations.

Other researchers have used neuroimaging procedures and magnetic brain stimulation to study the effects of passive movement training on sensorimotor brain networks. The neuroimaging studies have identified changes associated with passive 
movements in primary sensorimotor cortex (including both M1 and SI) and SMA in healthy individuals (Carel et al., 2000), and in SMA, prefrontal cortex, contralesional inferior parietal lobule, second somatosensory cortex, and ventral premotor areas in patients with subcortical stroke (Dechaumont-Palacin et al., 2008). Stroke patients also show increased activity with passive movement training bilaterally in premotor areas, inferior parietal cortex, and contralateral precentral gyrus (Nelles et al., 2001). In related studies using transcranial magnetic stimulation, passive wrist movement enlarged the map of the cortical representation of forearm muscles (Lewis and Byblow, 2004; Macé et al., 2008). As a group, these studies document plasticity in both sensory and motor cortices as a result of passive movement training and, in conjunction with the present finding, point to the involvement of motor areas of the brain in perceptual learning. The present work extends these findings by linking changes in sensorimotor networks to behavioral measures of learning, by using measures of connectivity in the resting brain to control for confounds arising from differences in task execution between scans, and by explicitly identifying changes in motor-related networks when the effects of activity in somatosensory networks are parceled out.

Overall, we find that perceptual learning results in a continuum of changes in connectivity in all levels of the sensorimotor network. This includes changes in motor areas of the brain that are independent of activity in the somatosensory network. In the study by Vahdat et al. (2011), we found that the perceptual changes that occur in association with motor learning likewise recruit a network involving PMv, SII, and SMA. Together with the results of the present study, these demonstrations suggest that in naturalistic situations where somatosensory and motor learning co-occur, a portion of the change in motor areas of the brain that occurs in learning skilled movements is actually a byproduct of perceptual learning.

\section{References}

Beckmann CF, Jenkinson M, Smith SM (2003) General multilevel linear modeling for group analysis in FMRI. Neuroimage 20:1052-1063. CrossRef Medline

Buckner RL, Andrews-Hanna JR, Schacter DL (2008) The brain's default network: anatomy, function, and relevance to disease. Ann N Y Acad Sci 1124:1-38. CrossRef Medline

Carel C, Loubinoux I, Boulanouar K, Manelfe C, Rascol O, Celsis P, Chollet F (2000) Neural substrate for the effects of passive training on sensorimotor cortical representation: a study with functional magnetic resonance imaging in healthy subjects. J Cereb Blood Flow Metab 20:478-484. Medline

Charlton CS, Ridding MC, Thompson PD, Miles TS (2003) Prolonged peripheral nerve stimulation induces persistent changes in excitability of human motor cortex. J Neurol Sci 208:79-85. CrossRef Medline

Conforto AB, Kaelin-Lang A, Cohen LG (2002) Increase in hand muscle strength of stroke patients after somatosensory stimulation. Ann Neurol 51:122-125. CrossRef Medline

Darainy M, Vahdat S, Ostry DJ (2013) Perceptual learning in sensorimotor adaptation. J Neurophysiol 110:2152-2162. CrossRef Medline

Dechaumont-Palacin S, Marque P, De Boissezon X, Castel-Lacanal E, Carel C, Berry I, Pastor J, Albucher JF, Chollet F, Loubinoux I (2008) Neural correlates of proprioceptive integration in the contralesional hemisphere of very impaired patients shortly after a subcortical stroke: an FMRI study. Neurorehabil Neural Repair 22:154-165. CrossRef Medline

de Lafuente V, Romo R (2006) Neural correlate of subjective sensory experience gradually builds up across cortical areas. Proc Natl Acad Sci U S A 103:14266-14271. CrossRef Medline

Forner-Cordero A, Steyvers M, Levin O, Alaerts K, Swinnen SP (2008) Changes in corticomotor excitability following prolonged muscle tendon vibration. Behav Brain Res 190:41-49. CrossRef Medline
Fox MD, Raichle ME (2007) Spontaneous fluctuations in brain activity observed with functional magnetic resonance imaging. Nat Rev Neurosci 8:700-711. CrossRef Medline

Hernández A, Nácher V, Luna R, Zainos A, Lemus L, Alvarez M, Vázquez Y, Camarillo L, Romo R (2010) Decoding a perceptual decision process across cortex. Neuron 66:300-314. CrossRef Medline

Kaelin-Lang A, Sawaki L, Cohen LG (2005) Role of voluntary drive in encoding an elementary motor memory. J Neurophysiol 93:1099-1103. CrossRef Medline

Lametti DR, Nasir SM, Ostry DJ (2012) Sensory preference in speech production revealed by simultaneous alteration of auditory and somatosensory feedback. J Neurosci 32:9351-9358. CrossRef Medline

Lewis GN, Byblow WD (2004) The effects of repetitive proprioceptive stimulation on corticomotor representation in intact and hemiplegic individuals. Clin Neurophysiol 115:765-773. CrossRef Medline

Lindberg P, Schmitz C, Forssberg H, Engardt M, Borg J (2004) Effects of passive-active movement training on upper limb motor function and cortical activation in chronic patients with stroke: a pilot study. J Rehabil Med 36:117-123. CrossRef Medline

Lotze M, Braun C, Birbaumer N, Anders S, Cohen LG (2003) Motor learning elicited by voluntary drive. Brain 126:866-872. CrossRef Medline

Ma L, Wang B, Narayana S, Hazeltine E, Chen X, Robin DA, Fox PT, Xiong J (2010) Changes in regional activity are accompanied with changes in inter-regional connectivity during 4 weeks motor learning. Brain Res 1318:64-76. CrossRef Medline

Macé MJ, Levin O, Alaerts K, Rothwell JC, Swinnen SP (2008) Corticospinal facilitation following prolonged proprioceptive stimulation by means of passive wrist movement. J Clin Neurophysiol 25:202-209. CrossRef Medline

Marrelec G, Krainik A, Duffau H, Pélégrini-Issac M, Lehéricy S, Doyon J, Benali H (2006) Partial correlation for functional brain interactivity investigation in functional MRI. Neuroimage 32:228-237. CrossRef Medline

Nelles G, Jentzen W, Jueptner M, Müller S, Diener HC (2001) Arm training induced brain plasticity in stroke studied with serial positron emission tomography. Neuroimage 13:1146-1154. CrossRef Medline

Ostry DJ, Darainy M, Mattar AA, Wong J, Gribble PL (2010) Somatosensory plasticity and motor learning. J Neurosci 30:5384-5393. CrossRef Medline

Penhune VB, Doyon J (2005) Cerebellum and M1 interaction during early learning of timed motor sequences. Neuroimage 26:801-812. CrossRef Medline

Pleger B, Foerster AF, Ragert P, Dinse HR, Schwenkreis P, Malin JP, Nicolas V, Tegenthoff M (2003) Functional imaging of perceptual learning in human primary and secondary somatosensory cortex. Neuron 40:643653. CrossRef Medline

Romo R, de Lafuente V (2013) Conversion of sensory signals into perceptual decisions. Prog Neurobiol 103:41-75. CrossRef Medline

Romo R, Salinas E (2003) Flutter discrimination: neural codes, perception, memory and decision making. Nat Rev Neurosci 4:203-218. CrossRef Medline

Rosenkranz K, Rothwell JC (2012) Modulation of proprioceptive integration in the motor cortex shapes human motor learning. J Neurosci 32: 9000-9006. CrossRef Medline

Stefan K, Kunesch E, Cohen LG, Benecke R, Classen J (2000) Induction of plasticity in the human motor cortex by paired associative stimulation. Brain 123:572-584. CrossRef Medline

Stefan K, Kunesch E, Benecke R, Cohen LG, Classen J (2002) Mechanisms of enhancement of human motor cortex excitability induced by interventional paired associative stimulation. J Physiol 543:699-708. CrossRef Medline

Vahdat S, Darainy M, Milner TE, Ostry DJ (2011) Functionally specific changes in resting-state sensorimotor networks after motor learning. J Neurosci 31:16907-16915. CrossRef Medline

Vahdat S, Maneshi M, Grova C, Gotman J, Milner TE (2012) Shared and specific independent components analysis for between-group comparison. Neural Comput 24:3052-3090. CrossRef Medline

Wong JD, Kistemaker DA, Chin A, Gribble PL (2012) Can proprioceptive training improve motor learning? J Neurophysiol 108:3313-3321. CrossRef Medline 